\title{
Mesoporous Catalyst Co-SBA-15 Catalytic Oxidation of 2-Methyl Pyridine to 2-Picolinic Formic Acid
}

\author{
Shan Jiongchen ${ }^{\mathrm{a}}$, Kong Fandong ${ }^{\mathrm{b}}$, Cao Yaqi ${ }^{\mathrm{c}}$, Tang Zhuoran ${ }^{\mathrm{d}}$, Yin Xin ${ }^{\mathrm{e}}$, Zhao Huiting , \\ Ling Aixia ${ }^{\text {, * }}$ \\ Department of Pharmacy, Jining Medical University, Rizhao, China \\ ashanjiongchen@aliyun.com, bjykfd@126.com, ㄷaoyaqi@163.com, ${ }^{\mathrm{d}} 409862257 @ q q . c o m$, \\ e1070349905@qq.com, ${ }^{\mathrm{f}} 1966008986 @ q q . c o m,{ }^{g}$ lingaixia@163.com \\ *Corresponding author
}

Keywords: Mesoporous Catalyst, Catalytic Oxidation, Co-SBA-15

\begin{abstract}
The synthesis rate of 2- picolinic formic acid catalyzed by 2-methyl pyridine was studied to investigate the activity of mesoporous catalyst. The purpose is to prove that the mesoporous catalyst used is a high performance heterogeneous catalyst. It was found that the amount of catalyst, reaction time, temperature and other factors would influence the reaction process. These factors were controlled to maximize the reaction activity.
\end{abstract}

\section{Introduction}

2-picolinic formic acid is a white crystalline powder. Molecular formula is C6H5NO2 (molecular weight 123.11), melting point (boiling point) is $136-139{ }^{\circ} \mathrm{C}$. It can be dissolved in hot water and ethanol. It can be used not only for the preparation of drugs, but also for the food feed additives and dyes ${ }^{[1-3]}$. The traditional chemical oxidation method can be used to prepare 2-picolinic acid acid with 2-methylpyridine ${ }^{[4]}$. Wang Zhide et al. ${ }^{[5]}$ obtained 2-picolinic formic acid as a finished product by catalyzing 2-methyl pyridine in the liquid phase, and the yield was about $85 \%$. However, this method is less and less common in industry because of the high pollution, low yield and difficulty in separating products. Compared with the liquid phase catalysis, the gas phase catalytic oxidation method $^{[6]}$ requires a higher temperature, and its advantage is that the product is easy to be separated. The optimum conditions for the synthesis of 2-pyridine by 2-methyl pyridine using mesoporous materials Co-SBA-15 were studied, and the rate and yield of the reaction were measured from the amount, time and temperature of the catalyst.

\section{Experimental Method}

\subsection{Preparation of Co-SBA-15 Mesoporous Catalysts}

On the basis of reference ${ }^{[1]}$, $1 \mathrm{~g}$ hexadecyl trimethyl ammonium bromide(CTAB) was dissolved in deionized water to form a homogeneous solution. About $15 \mathrm{ml} 37 \%$ hydrochloric acid was then added and the mixture was ultrasonicated for 1 hour. Afterwards, under vigorous stirring, $4.47 \mathrm{ml}$ silicon source-tetraethyl orthosilicate (TEOS) were successively added. After it was completely dissolved, weighed proportionate $\mathrm{Co}\left(\mathrm{NO}_{3}\right)_{2} \cdot 6 \mathrm{H}_{2} \mathrm{O}$ and dissolved in deionized water, dropped into the above solution, magnetic stired at $40^{\circ} \mathrm{C}$ for 24 hours. Then transfer the obtained mixed solution to crystallization in tetrafluoroethylene bottle. The reaction was carried out in a $90^{\circ} \mathrm{C}$ water bath for 1 day and then slowly cooled naturally. The obtained solution was ultrafiltration and washed several times with ethanol. Finally, the white powder is obtained at room temperature by natural cooling and drying. Put it into the muffle furnace for 6 hours. The co-sba- 15 can be got, the surfactant CTAB is removed.

\subsection{Catalytic Oxidation Reaction}

Under the oil bath of $80 \mathrm{C}$, the catalyst of $55 \mathrm{mg}, 15 \mathrm{~mL}$ acetic acid solvent and $0.59 \mathrm{~g}$ of 2-methyl 
pyridine were added to 50mL two necked flask, until the mixture was mixed uniform then ultrasonic 20min. When the reaction starts, added 30 percent peroxide solution to the reaction system about a drop of 5 seconds, then under the condition of condensing reflux and following the magnetic stirring about 6h. Dilute the mixture with water after cooling., then the product was analyzed with a high efficiency chromatograph., The mixture was diluted with water, after it was cooled, then filtered, and the product was analyzed with a high efficiency chromatograph.

\section{Results and Discussions}

\subsection{XRD Analysis of Co-SBA-15 Mesoporous Catalysts}

The Low Angle X-Ray Diffraction spectrum and the High Angle X-Ray Diffraction spectrum of the sample Co-SBA-15 are shown in Fig.1.1.There are four peaks in the low angle region between $2 \theta \sim 0.5-7.00$, which indicates that the obtained material has hexagonal crystal pore structure. That is the characteristic peak of MCM-41, and the essence of Co-SBA-15 is the MCM-41 synthesized under acidic condition.

Through the intensity of these four peaks (HKL=100,110,200,210), the mesoporous material obtained is a porous structure with high permeability and well- ordering. From the figure 1.1, we get the amorphous material. It is important to emphasize that the test results indicate that there is no Co in the sample, which indicates that $\mathrm{Co} 2+$ ion has been highly dispersed or bonded to the silicon structure of SBA-15.
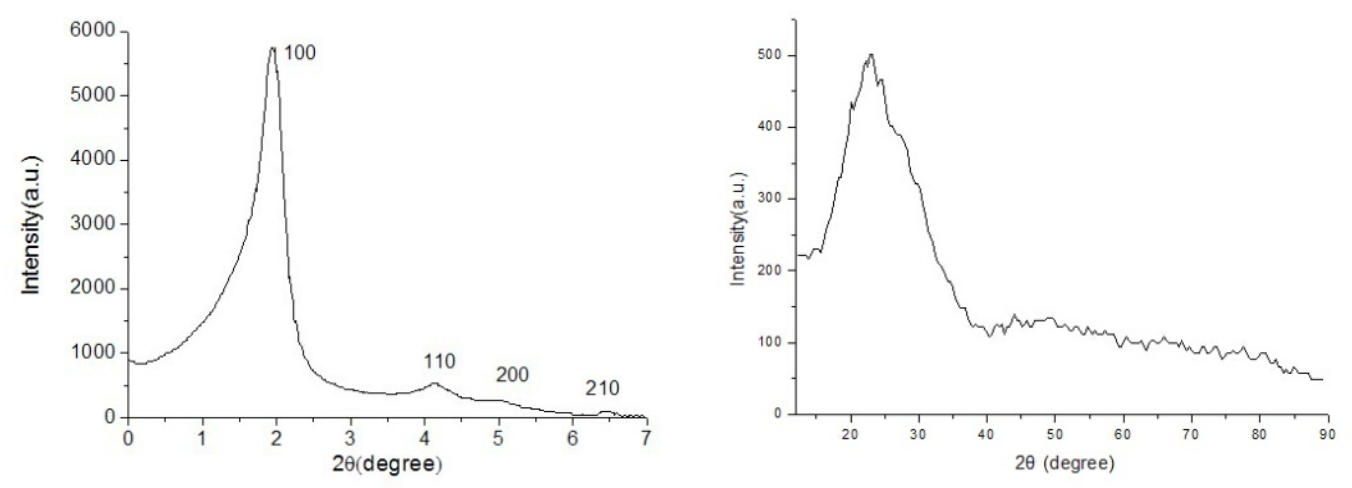

Figure 1 XRD of Co-SBA-15.

\subsection{Study on the Catalytic Performance of Co-SBA-15 Mesoporous Catalyst in the Synthesis of 2-Picolinic Formic Acid}

The experimental results show that 2- picolinic formic acid is the most important oxidation product when using Co-SBA-15 as catalyst to catalyze 2-methyl pyridine, and a small amount of 2pyridine formaldehyde, pyridine. In this experiment, we use acetic acid as a solvent, and discuss the influence of reaction temperature, reaction time, catalyst and other factors on the reaction.

\subsubsection{Effect of temperature on the reaction}

The effect of temperature on this reaction can be seen from Figure 1.2. The selectivity of 2picolinic formic acid will increase first and then decrease with the increase of temperature. In especial the increase was evident in $353 \mathrm{~K} \sim 366 \mathrm{~K}$. The conversion rate of 2- methyl pyridine increased with the increase of temperature, but it does not change much when the temperature was more than 366K. It may be because with the increase of temperature, the decomposition of hydrogen peroxide and the volatilization of glacial acetic acid are accelerated. Therefore, the temperature was controlled at 366K for the best reaction temperature in this experiment. 


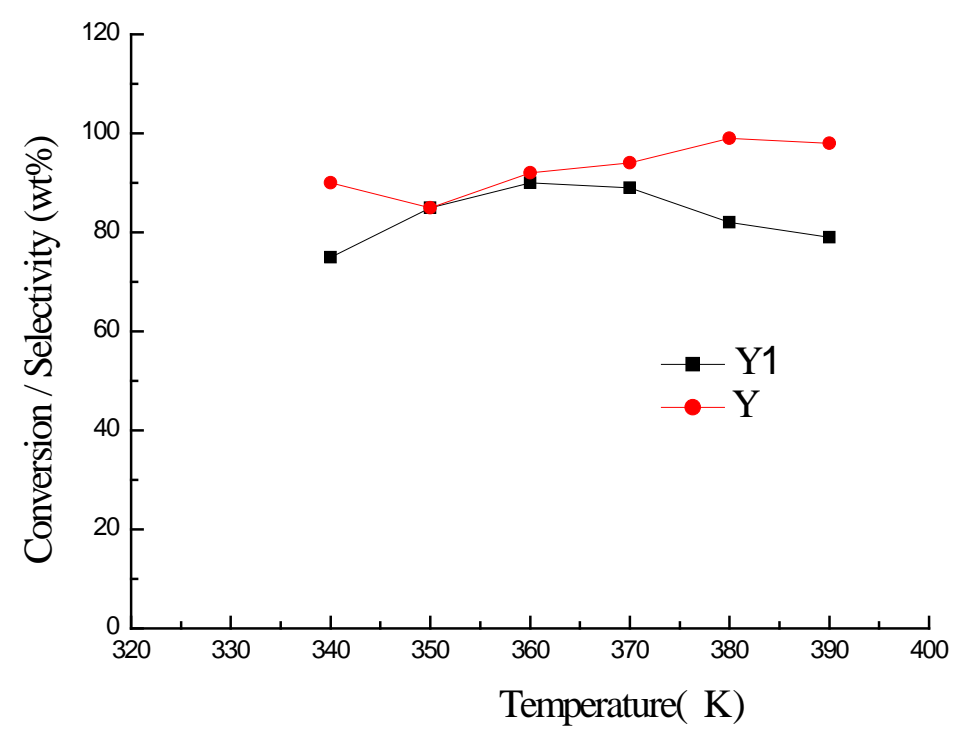

Figure 2 The effect of reaction temperature on the selectivity and conversion of Co-SBA-15 reaction.

\subsubsection{Effect of time selection on the reaction}

The results of the experiment were also investigated in $4-12 \mathrm{~h}$, and the results were shown in figure 1.3. It can be seen from the figure that the conversion rate of 2-methyl pyridine increases gradually over time, and the change is not too much. But when it gets to 10h, the selectivity of 2- picolinic formic acid reaches the maximum. Therefore, the optimal reaction time of this experiment is $10 \mathrm{~h}$.

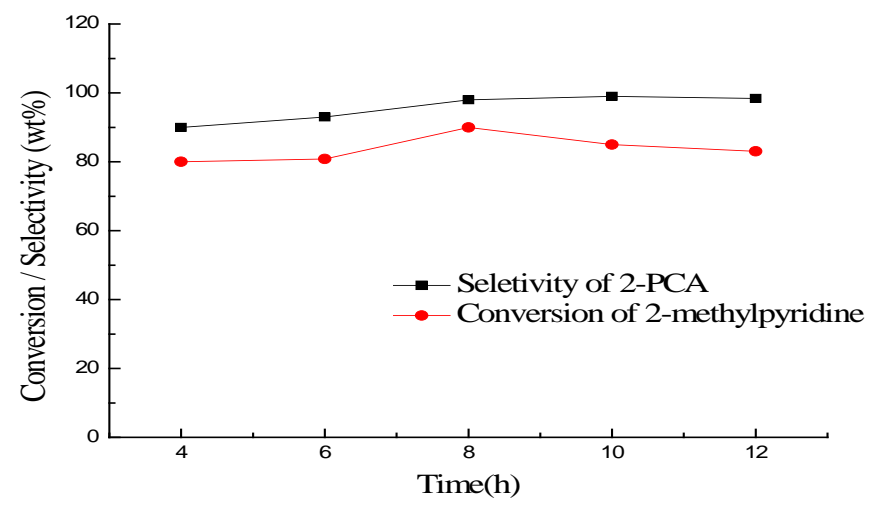

Figure 3 The effect of reaction time on the selectivity and conversion of Co-SBA-15 reaction.

\subsubsection{Effect of the Amount of Catalyst on the Reaction}

The effect of the addition of Co-SBA-15 is examined on the catalytic reaction under the same conditions. The catalysts were studied with $10 \mathrm{mg}, 20 \mathrm{mg}, 30 \mathrm{mg}, 40 \mathrm{mg}, 55 \mathrm{mg}$ and $100 \mathrm{mg}$, respectively. The results are shown in Fig. 1.4. Experiments showed that the conversion of 2-methylpyridine and the selectivity of 2- picolinic formic acid were increased when using 55mg catalyst. When 100mg catalyst was added, the conversion rate of 2-methyl pyridine increased but the selectivity of 2-pyridine carboxylic acid decreased. Therefore, it is most appropriate to select 55mg catalyst for the experiment. 


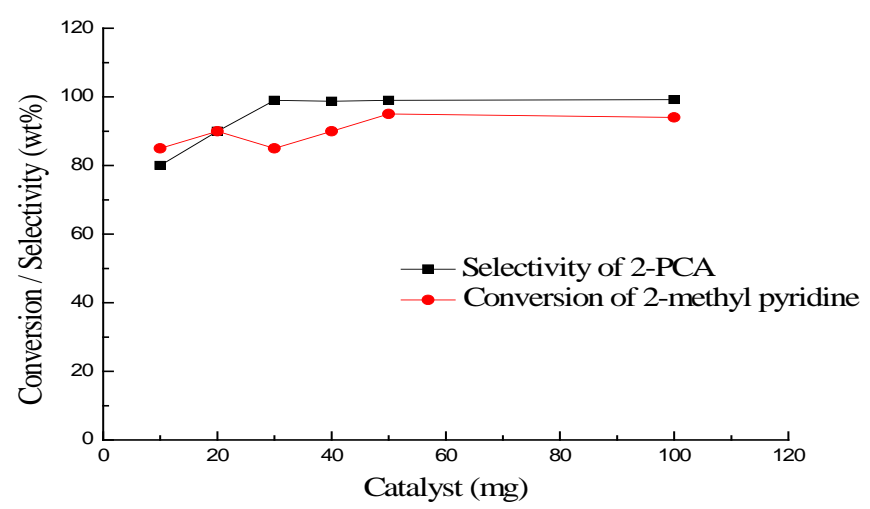

Figure 4 The effect of the amount of catalyst on the selectivity and conversion of Co-SBA-15 reaction.

\section{Conclusions}

From the results of the characterization, it can be found that the Co-SBA-15 prepared is a kind of micro-mesoporous material, which is a white 2-d spherical superfine mesoporous material. Catalytic oxidation activity results indicate that when 2-methyl pyridine was $0.57 \mathrm{~g}$ Catalyst dosage of $55 \mathrm{mg}$, oxidant dosage is $7 \mathrm{ml}$, with $15 \mathrm{ml}$ ice acetic acid solvent, reaction temperature of $366 \mathrm{~K}$, reaction time is $10 \mathrm{~h}$, 2-methyl pyridine conversion rate of $95.5 \%$, the selectivity of 2- picolinic formic acid is $90.7 \%$.

\section{Acknowledgements}

This work is financially supported by National Innovation Training Program of Ministry for College Students (No.201610443026), Undergraduate Innovation Training Program of Jining Medical University (No.CX2016026), Shangdong Provincial Natural Science Foundation (ZR2017LB014), and the National Innovation Training Program for College Students (No. 201510443002)

\section{References}

[1] Xu Kexun. Manual of fine organic chemical materials and intermediate materials[M]. Beijing: Chemical Industry Press,1999:57-68.

[2] Chen Guangrong. Encyclopedia of chemical industry[M]. Beijing: Chemical Industry Press, 1990:557-584.

[3] Liu Xiaohua, Du xi, and Li Xian. Progress in preparation of pyridine formic acid. Chemical research and application,2003,15(2):161-164.

[4] Wang Haitang, Wang yongxin, Pan Zhiquan. A new method for synthesis of 2-pyridine formic acid. Hubei chemical,2001, (2): 26-27.

[5] Wang Zhide. The present situation and prospect of nicotinic acid and thalidomide. Natural gas chemical, 1992, (4):4749.

[6] Li G X, Wang X P, Qin F. Preparetion of nicotinic acids by air oxidation of 3-picoline. Yiyao gongye, 1984, (1):1- 5. 